\title{
Characterization of the proton pulsed beam at CMAM
}

\author{
S. Viñals ${ }^{1}$, D. Sánchez-Parcerisa ${ }^{2}$, L.M. Fraile ${ }^{2}$, S. España ${ }^{2}$, G. García ${ }^{1}$, M. García-Díaz ${ }^{2}$, V. Sánchez- \\ Tembleque $^{2}$, J.M. Udías ${ }^{2}$ \\ ${ }^{1}$ Centro de Micro-Análisis de Materiales, Madrid, Spain \\ ${ }^{2}$ Grupo de Física Nuclear, Universidad Complutense, Madrid, Spain \\ silvia.vinnals@uam.es
}

\begin{abstract}
In this paper, the technicalities performed to obtain a pulsed beam at the CMAM facility will be explained. The pulsed beam has been characterized with an $8 \mathrm{MeV}$ proton beam, using an existing equipment at CMAM: two pairs of electrostatic plates (RASTER) that deflect the beam, commonly used for homogeneous irradiation of large areas. A pulsed beam is used in many areas such as nuclear physics, material science and, in particular, for proton-therapy medical studies. Rectangular and pyramidal functions have been used to generate different pulses and characterize the response of the RASTER. The results point out that the pulses obtained are suitable for preclinical protontherapy studies in the FLASH regime, which consists on fractionating the dose in time with short and intense pulses. The set-up for the characterization has been a function generator and a Si-PM outside the chamber.
\end{abstract}

Keywords -Pulsed Beam in air, Electrostatic Deflection.

\section{INTRODUCTION}

\section{A. CMAM: The facility}

The Centro de Micro-Análisis de Materiales (CMAM) is one of the two ion accelerators research centers in Spain. It belongs to the Universidad Autónoma de Madrid (UAM) and the building that hosts the laboratory is at the university campus.

The equipment of the facility consists of an electrostatic ion accelerator with a maximum terminal voltage of $5 \mathrm{MV}$ and six beam-lines dedicated to various application areas such as the analysis and modification of materials, the study of the nuclear reactions or archaeometry studies. The accelerator is unique in Spain and singular in the European context [1]. It was installed in the early 2000 s and has been in regular operation ever since.

The accelerator, built by High Voltage Engineering Europe (HVEE), is of the tandem type with a Cockroft-Walton acceleration system. It is provided with two sources: a plasma source for gaseous substances and a sputtering source for obtaining any element from $\mathrm{H}$ to $\mathrm{U}$ from a solid target.

The accelerator feeds up six beam-lines after the bending magnet at different angles: the standard multi-purpose line which ends in the internal micro-beam line, the time of flight line, the implantation line, the external micro-beam line and the nuclear physics line. For a more detailed description of each line, see Ref. [2]. Figure 1 shows a scheme of the accelerator with its spatial distribution (a) and a picture of the accelerator hall from the point of view of the end of the experimental lines (b). There are two lines where the beam can be extracted from vacuum to air: the implantation line (IMP) and the external micro-beam line (EuB). Until now, the former has been devoted to the implantation or irradiation of large area samples for material modification and the latter has been focused onto archaeometry studies. Now, a new project has started to use these two lines for proton-therapy preclinical studies.
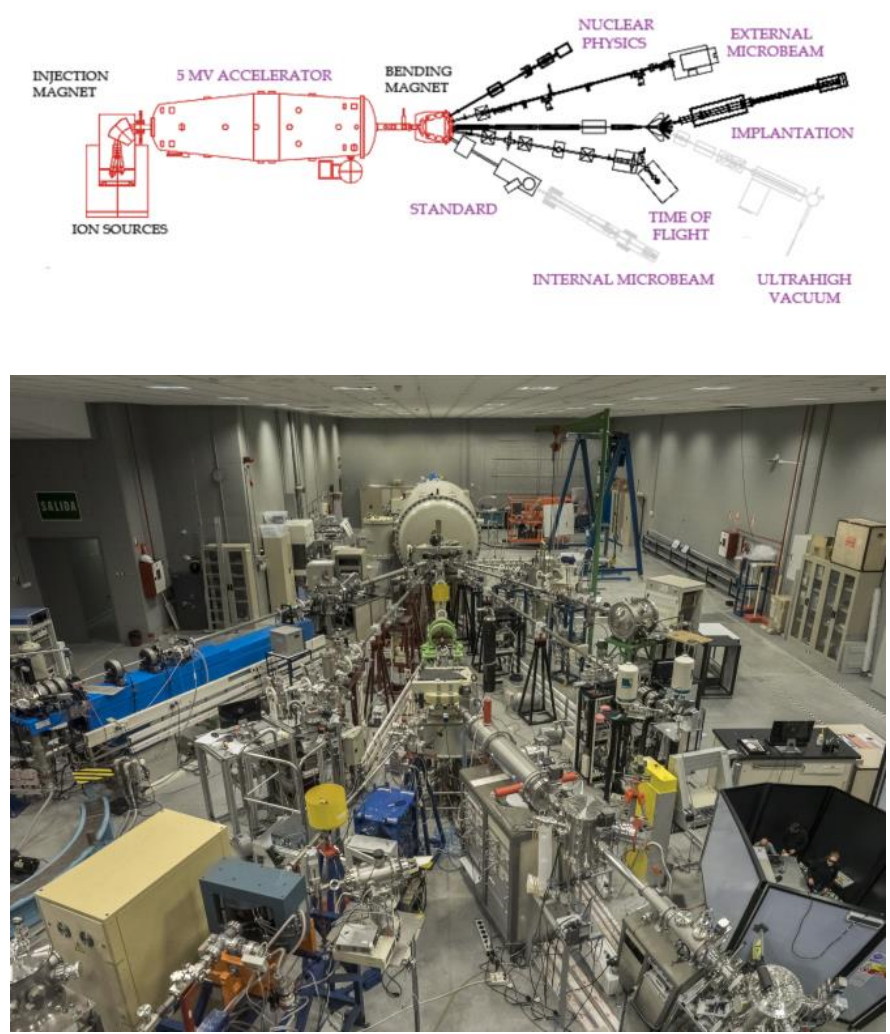

Fig. 1. (a) Scheme of the accelerator hall with the experimental lines identified. (b) Picture of the CMAM accelerator hall from point of view of the end of the experimental lines.

Nowadays, the facility has requested funds to upgrade the accelerator hall installing a pulsed light-ion source. It will be installed in the low energy part of the accelerator and will feed up the six experimental lines. However, this improvement will not be useful until a couple of years. In the following sections, the paper will describe how the facility has taken benefit of one 
of the existing elements in the center for pulsing the beam in air in one of the lines, the IMP, and thus opening the opportunity to some preliminary experiments, to be further developed once the upgrade of the sources will be fully implemented.

\section{B. The Implantation line}

The line is placed at the $-20^{\circ}$ exit port after the second high energy switching magnet, allowing experiments in a wide range of ions and ionic species with a reduced beam-current loss. The line has a total length of 6 meters and ends in a large irradiation vacuum chamber that offers the possibility of extracting the beam in air through various slits equipped with suitable output windows.

The main aim of this beam-line is to perform homogeneous implantations or irradiations in large areas (up to several $\mathrm{cm}^{2}$ ). For this purpose, the line has a HVEE electrostatic scanner consisting of four independently polarized plates, designed specifically for the CMAM facility. This system is capable of scanning Hydrogen of $10 \mathrm{MeV}$ at a size of $100 \times 100 \mathrm{~mm}^{2}$ when the two plate sets are used at the same time scanning at incommensurate frequencies, so that the scanning trajectory of the beam spot on the sample plane becomes ergodic. This is the most restrictive case and the one that will be considered for the purpose of this paper. Until now, this system called RASTER has been used merely for scanning the beam for irradiating in the mentioned large areas; nevertheless, it can open the door to obtain external pulsed beams.

\section{The RASTER}

The system used to pulse the beam is composed by two pairs of fast beam deflection electrostatic plates which accept an input signal of a maximum of $10 \mathrm{~V}$ in each of the plates. The voltage of the input signal is converted to $\mathrm{kV}$ to deflect the beam.

In the deflection process, the high voltage is applied to the electrode of the line to cast the beam. Then, the electric field is produced to deflect the beam. By modifying the conditions of the voltage induced in each of the plates, the deflection can follow an ergodic pattern, used for homogeneous irradiations of large areas, or deflect the beam at desired.

The deflection from the origin point (D) produced by one electrostatic plate is calculated following equation (1)

$$
D=\frac{L \cdot \delta \cdot E_{d}}{2 d \cdot E_{a}}
$$

where $L$ is the length of the line from the beginning of the electrostatic plates to the irradiation point, $\delta$ the length of the deflection plates, $E_{d}$ the voltage difference applied to the parallel plates, $d$ the distance between the plates and $E_{a}$ the energy divided by the charge state of the beam when passing through the plates.

The characteristics of the CMAM's RASTER are: $\delta=447.7$ $\mathrm{mm}, d=132.5 \mathrm{~mm}$ and $L=6 \mathrm{~m}$.

\section{Pulsed ion-beam applications}

The capability of pulsing an ion beam in a facility opens the door for many applications such as material science, nuclear physics and medical physics.

For material science, which is one of the main research activities developed at CMAM, a pulsed ion-beam has been proved to be effective in annealing of ion implantations damage, in formation of $\mathrm{p}-\mathrm{n}$ junctions in a single step doping process or in silicide formation as examples [3]. Moreover, the pulsed beam can be synchronized with the femtosecond (fs) pulsed laser installed at CMAM allowing to explore what is called soft materials [4].

In the nuclear physics case, being available to adjust the time structure of the pulsed beam will enhance the statistics by synchronizing the electronic acquisition with the beam as well as allow for performing time of flight spectroscopy. This will be a useful approach to reduce the dead-time of the acquisition systems and therefore obtain better statistics. Also, it will allow to study the nuclear structure through time of flight measurements [5].

Finally, in the medical physics field, a pulsed proton beam is of strong interest in the radiobiology and radiotherapy studies. In the last years, a new approach to irradiate tumors has been topic of research around the world: the FLASH treatment, where the given dose is fractionated in time with short and intense pulses. This will be explained in detail in the following subsection as the characterization presented in this document has the final objective of being used for this type of experiments.

\section{E. Proton-therapy in FLASH regime}

Proton-therapy is known as the radiotherapy technique for cancer treatment that uses proton beams for producing the damage. The main difference between the conventional radiotherapy treatments with X-Ray or electrons and the treatment with protons relays on how the deposition of energy is produced by each particle. In the case of the protons, most of the energy is delivered at the Bragg's peak region, which is a pronounced peak at a certain depth where the protons are completely stopped. One of the main advantages of the proton-therapy is that after the Bragg's peak, no dose is delivered assuring that healthy tissues behind the tumors are not radiated. The delivered dose curve for photons (or X-Ray) and electrons is wider at the superficial region and attenuates exponentially. Figure 2 presents the different curves of energy deposition for photons (blue), electrons (red) and protons (green).

Recent works in radiotherapy have raised interest in studying the outcome of very high dose rates, the so-called FLASH irradiations (>40 Gy/s), which are able to considerably reduce side effects compared to standard dose rates $(\sim 5 \mathrm{cGy} / \mathrm{s})$. It is based on delivering ultra-high dose-rates inducing differential biological effects between normal and tumoral tissues and 
reducing the radiation-induced side effects [6-7]. The effectiveness of the FLASH-RT compared with the conventional radiotherapy plan has been demonstrated with electrons [8] and X-Ray [9]. Recently, its feasibility has been demonstrated with flash proton irradiation [9]. For the FLASH$\mathrm{RT}$, high intensities in short periods of time are required.

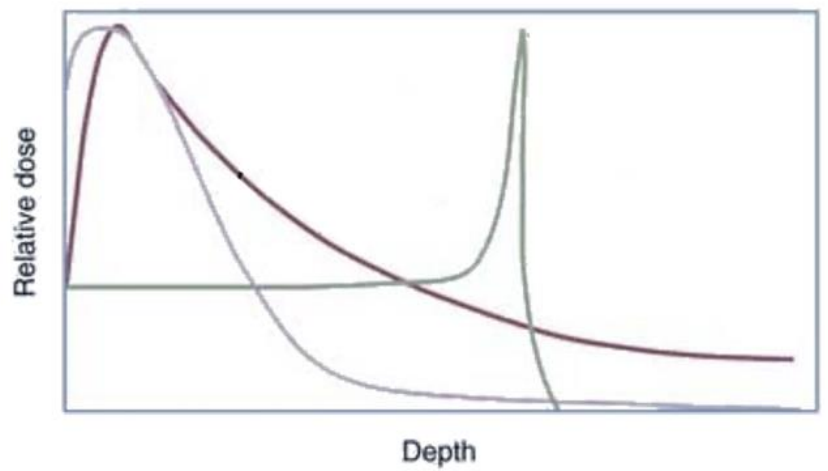

Fig. 2. Relative dose deposition as a function of the interaction depth for photons (red), electrons (blue) and protons (green).

\section{Characterization of the PUlses}

\section{A. Experimental conditions}

The experiment aimed at the characterization of the pulsed beam for proton-therapy pre-clinical studies. The beam used was a proton-beam of $8 \mathrm{MeV}$ and $5 \mathrm{~mm}$ of diameter. These conditions were kept fixed during the experiment. The chamber was prepared to reduce the possible activation of the elements that interact with the beam by covering them with Ta foils of $250 \mu \mathrm{m}$. The beam was visible thanks to 2 arrays of scintillator pixels of $\mathrm{SiO}_{2}$ that emit blue light as a response to the interaction with the beam. With the RASTER, the beam is moved along the silicon scintillator and only when it crosses the aperture, the beam exits the vacuum chamber and irradiates the sample placed in air. In Fig. 3 a picture of a $\mathrm{SiO}_{2}$ placed inside of the chamber is shown, where the two blue spots are the positions where the beam reaches its maximum deflection and stays most of the time.

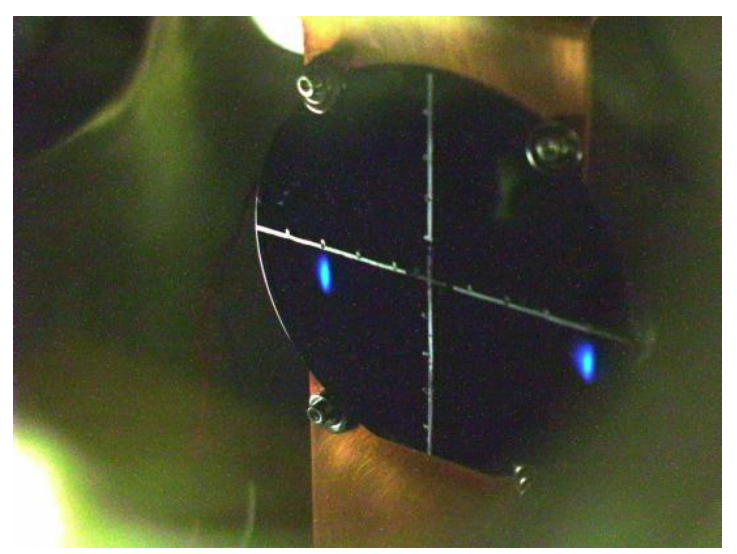

Fig. 3. Picture of a $\mathrm{SiO}_{2}$ scintillator placed inside the IMP chamber. The two blue spots are the positions where the beam is most of the time.
Once the beam is extracted outside the vacuum chamber through the aperture and output window, it is characterized with a setup that consists of a CeGAG scintillator $(1 \times 1 \times 3 \mathrm{~cm})$ coupled to a S13360-6075CS SiPM from Hamamatsu. The signal is read directly with a digital oscilloscope, using the Picospe 6403D, with 8 bit resolution and a bandwidth of 350 $\mathrm{MHz}$.

The function generator was connected to one of the $\mathrm{X}$-axis deflection plates, moving the beam to the right. From the RASTER, the voltage signal produced was sent to the Picoscope to be processed and used as a trigger for the time signal. Due to the capacitance of the plate, a minimum time is needed to charge the plate. The minimum time for charge the electrostatic plate with $7 \mathrm{kV}$ is $20 \mu \mathrm{s}$ and, for rectangular functions, the resultant signal at RASTER will have a raise time. Different functions were tested in order to obtain the best conditions, optimizing the time of the pulses and avoiding the overshooting effect produced by forcing the plate to be charged/discharged faster than the time required by the capacitor.

\section{B. Results}

During the experiment, other functions were tested such as exponential rise, however, the function that best matches with the objectives of pulsing a beam for a FLASH proton-therapy treatment was the pyramidal one. In Table 1 it is detailed the parameters of the two functions analyzed: the rectangular and the pyramidal.

\begin{tabular}{ccc}
\hline \hline Function & Amplitude & Width \\
\hline Rectangular & $7 \mathrm{~V}$ & $130 \mu \mathrm{s}$ \\
Pyramidal & $8 \mathrm{~V}$ & $120 \mu \mathrm{s}$ (ramp up)
\end{tabular}

Table 1. Parameters for the definition of the functions

Figure 4 summarizes the results of the experiment, comparing the pulse shape of the two functions studied. The first column is the input voltage of the RASTER generated with a function generator. The second, the output voltage from RASTER, which indicates how the plate has been charged. The third column is the signal detected on the Si-PM, which would be the pulse used to irradiate. Fig. 4a (first row) are the voltages for a rectangular pulse and Fig. $4 \mathrm{~b}$ (second row), the results for the pyramidal function. It can be seen that for the latter, the pulse is faster and the beam only exits the chamber once. When using the rectangular function, the beam exits twice from the chamber due to the overshoot. The discharge of the pyramidal function also produces an overshoot at the end of the pulse; however it does not affect the pulse signal detected on the Si$\mathrm{PM}$ as the beam only exits the chamber at the maximum voltage. 
Input RASTER
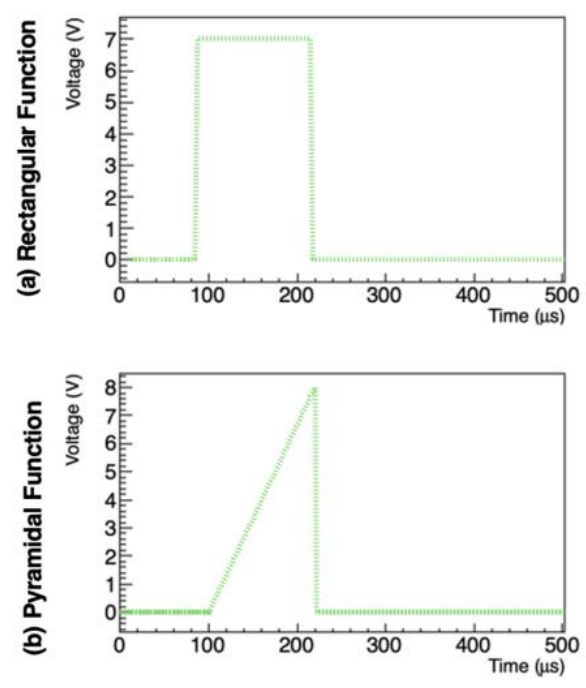

Output RASTER
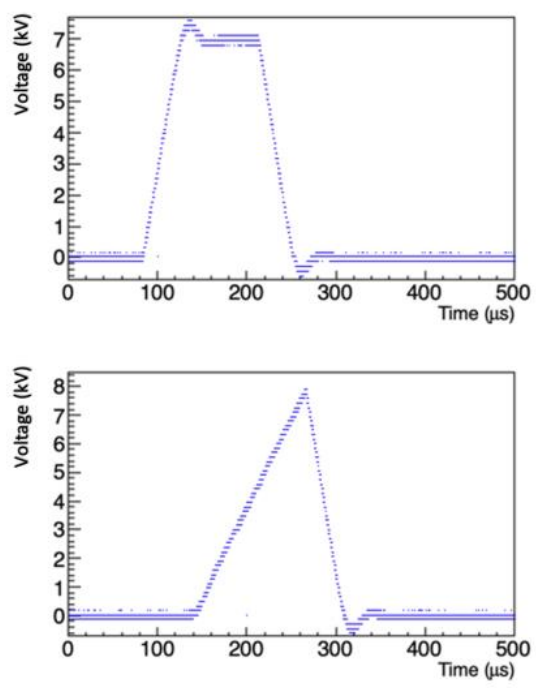

Si-PM signal
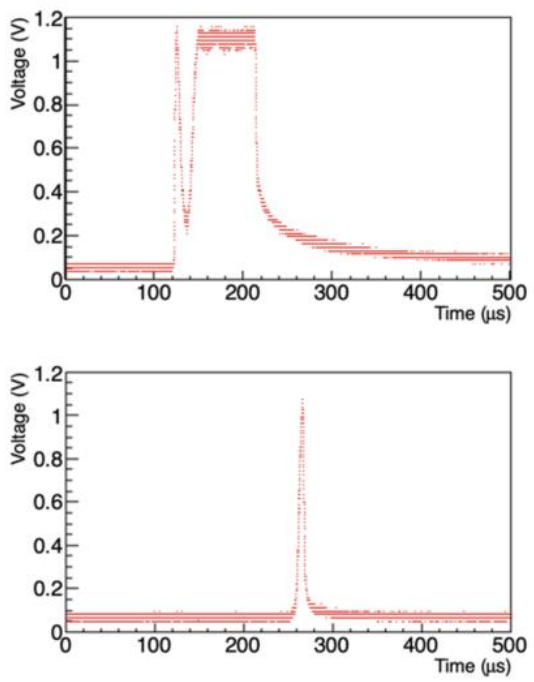

Fig. 4. Input, Output and Si-PM signal for two functions generated for pulsing the beam. First row (a) are the voltages obtained in each step for a rectangular function of $7 \mathrm{~V}$. The second row (b), for a pyramidal function of $8 \mathrm{~V}$. In green, the function generated used as input for the RASTER. In blue, the voltage induced on the RASTER's plate. In red, the signal detected in the Si-PM outside the chamber.

With the results obtained, the duration of the shortest pulse that can be obtained with this system has been determined: 10 $\mu \mathrm{s}$, using the pyramidal function. With the rectangular function, the length of the pulse can be modified but due to the overshoot effect, the pulse produced by a rectangular function will be difficult to characterize due to the fact that it has a double peak.

\section{Application of the pulses to a FLASH proton-therapy irradiation}

In a conventional proton-therapy treatment, the dose given in each session ranges from 3 to 9 Gy. For a FLASH protontherapy planning, the minimum beam intensity required with an $8 \mathrm{MeV}$ proto-beam is $40 \mathrm{pA}$. The beam operation conditions allow to increase the intensity up to $10 \mathrm{nA}$, which will produce a dose rate $(\dot{D})$ of $1000 \mathrm{~Gy} / \mathrm{s}$. These conditions are the most suitable operation conditions due to beam stability and activation thresholds.

Considering the beam conditions described above, to deliver a treatment dose ( $3 \mathrm{~Gy}$ ) in the FLASH regime with pulses of 10 $\mu \mathrm{s}$, the number of pulses required is 296 . Even though it seems a large number of pulses, the separation between the pulses can be less than $5 \mathrm{~ms}$, irradiating the sample in less than 2 seconds. Due to the shape of the signal detected on the Si-PM, it is not recommended to use the rectangular function, that will allow to produce longer pulses but with an overshoot difficult to quantify.

\section{CONCLUSIONS}

In this paper we present the characterization of the first pulsed beam in air obtained at the CMAM facility. The pulsing of the beam is obtained deflecting it using an electrostatic plate commonly used for homogeneous irradiations of large areas.
The characterization of the pulses has been done with a generator function, a Si-PM installed outside the irradiation chamber of the IMP line and a picoScope to register and trigger the voltage signals in each of the three steps: the input function, the output voltage at the electrostatic plates and the signal detected in the Si-PM.

Rectangular and pyramidal functions have been used to characterize the system. The former has the advantage of being able to modify at will the duration of the pulses by increasing the width of the signal. However, the pulse is not optimal as the beam exits the chamber twice per cycle. This effect is due to the overshoot produced by using a function that increases the voltage faster than the time that the capacitor requires to charge. With the pyramidal function, pulses of $10 \mu$ s are obtained, being suitable for many applications and, in particular, for protontherapy treatments in FLASH regime.

\section{ACKNOWLEDGMENT}

The authors want to thank the effort of the technician team of CMAM and the help provided by J. Olivares, V. Joco and F. Arias-Valcayo. 


\section{REFERENCES}

[1] D.J.W. Mous et al., "Performance and Applications of the first 5MV Tandetron at the University of Madrid." Proceedings CAARI 2002, AIP Conference Proceedings, August 26, 2003, Volume 680, Issue 1, pp. 999 1002.

[2] A. Redondo-Cubero et al., "Current status and future developments of the ion beam facility at the centre of micro-analysis of materials in Madrid" Eur. Phys. J. Plus, 2021, pp. 136-175

[3] J. Piekoszewski and J. Langner, "High intensity pulsed ion beams in material processing: Equipment and applications" in NIM B: Beam Interactions with Materials and Atoms, vol. 53, Issue 2, 1991, pp.148-160.

[4] Pavel Y. Apel, "Fabrication of functional micro- and nanoporous materials from polymers modified by swift heavy ions", Radiation Physics and Chemistry 159 (2019) 25-34.

[5] J. RD Copley and Terrence J. Udovic. "Neutron time-of-flight spectroscopy." Journal of research of the National Institute of Standards and Technology 98.1 (1993): 71.

[6] P. Montay-Gruel et al. "Irradiation in a flash: Unique sparing of memory in mice after whole brain irradiation with dose rates above $100 \mathrm{~Gy} / \mathrm{s}$." Radiotherapy and Oncology 124.3 (2017) 365-369.

[7] J. Bourhis et al. "Treatment of a first patient with FLASH-radiotherapy." Radiotherapy and Oncology 139 (2019): 18-22.

[8] P. Montay-Gruel et al. "X-rays can trigger the FLASH effect: Ultra-high dose-rate synchrotron light source prevents normal brain injury after whole brain irradiation in mice." Radiotherapy and Oncology 129.3 (2018): 582-588.

[9] E. Beyreuther et al. "Feasibility of proton FLASH effect tested by zebrafish embryo irradiation.” Radiotherapy and Oncology 139 (2019): 46- 50 . 